mortality, non-fatal myocardial infarction, referral/admission to hospital with CP) up to a minimum of 6 months after the index assessment were analysed. Typical, atypical and NACP were determined from CP characteristics. 622 patients without confirmed CAD were diagnosed with NACP. Following the 1st visit, 70 patients were recommended coronary angiograms (27 significant CAD, (26 revascularised, 1 treated medically), 7 refused) and 66 had myocardial perfusion scans (14 reversible/2 fixed defects). 62 (10\%) patients reached an end-point (2 cancer deaths, 11 non-fatal MI, 40 readmitted and 8 rereferred with CP, 1 nonhaemorrhagic stroke). Of these patients, 29 were previously investigated: 21 angiograms (10 significant CAD), 14 MPS (3 reversible/1 fixed defect). Investigations after reaching end-point showed 3 significant CAD (14 angiograms) and 1 reversible defect (5 myocardial perfsion scan). 14 others were not investigated further due to unequivocally negative exercise ECG. Only the presence of diabetes predicted the end-point (OR 5.21, 95\% CI 2.67 to $10.15, p<0.0001)$ in a multiple logistic regression analysis using age, gender, current smoking, total cholesterol $>6.47 \mathrm{mmol} / \mathrm{l}$ and diabetes as covariates.

Conclusion $47(7.6 \%)$ patient that are to be discharged without investigation, had significant coronary artery disease. Even with a short follow-up, $10 \%$ of these patients needed medical attention for suspected cardiovascular morbidity. It may be unreasonable to discharge patients describing NACP especially diabetics.

\section{CLINICAL OUTCOMES OF PATIENTS EXCLUDED FROM CARDIAC INVESTIGATION IN THE NICE GUIDELINES FOR CHEST PAIN OF RECENT ONSET}

doi:10.1136/heartjnl-2012-301877b.139

${ }^{1} \mathrm{C}$ Patterson, ${ }^{* 2} \mathrm{~N}$ Ahmed, ${ }^{3} \mathrm{E}$ Nicol, ${ }^{4} \mathrm{~L}$ Bryan, ${ }^{1} \mathrm{D}$ Bell. ${ }^{1}$ Imperial College, London, UK; ${ }^{2}$ Ealing Hospital, London, UK; ${ }^{3}$ Royal Brompton Hospital, London, UK; ${ }^{4}$ St Thomas' Hospital, London, UK

Introduction NICE guidance for the investigation of chest pain of recent onset ${ }^{1}$ recommends clinical assessment and risk stratification within a diagnostic algorithm. Patients with pain considered "nonanginal", and those with atypical/typical anginal pain but a likelihood of coronary artery disease $(\mathrm{CAD})<10 \%$ are not routinely recommended for cardiac investigation. This study sought to assess whether clinical outcomes support these patients being considered at low risk of CAD.

Methods 557 consecutive patients (50.4\% male; median age $55 \mathrm{yrs}$ ) attending rapid access chest pain clinics (RACPC) at two hospitals were risk stratified using NICE criteria. Frequency of admission with suspected angina, diagnosis of $\mathrm{CAD}$ and incidence of major adverse cardiac events (MACE: myocardial infarction (MI), cerebrovascular accident (CVA), emergency revascularisation or cardiac-related death) were compared for all risk categories at 6 months.

Results Of $360 / 557$ patients with "non-anginal" pain, 14 (3.9\%) were subsequently admitted with angina, 34 (9.4\%) were diagnosed with CAD, $3(0.8 \%)$ with MI and $2(0.6 \%)$ with CVA. This group accounted for $36.9 \%$ of all patients diagnosed with $\mathrm{CAD}$ and $38.5 \%$ of all patients with MACE. Of 10/557 patients with atypical/typical anginal pain and a likelihood of $\mathrm{CAD}<10 \%, 1(10 \%)$ was diagnosed with $\mathrm{CAD}$. None were admitted with angina or diagnosed with MACE. This group accounted for $1.1 \%$ of all patients diagnosed with $\mathrm{CAD}$

Conclusions This study suggests one in ten patients routinely excluded from cardiac investigation by the NICE algorithm have $\mathrm{CAD}$ and just over one in a hundred of them have a MACE episode. Although these patients are considered low risk, they account for one third of adverse cardiac events in patients attending RACPC.

\section{REFERENCE}

1. National Institute for Health and Clinical Excellence. CG95 Chest pain of recent onset assessment and diagnosis of recent onset chest pain or discomfort of suspected cardiac origin. NICE, 2010.

\section{ASSESSMENT OF PRE-TEST LIKELIHOOD OF CORONARY ARTERY DISEASE IN PATIENTS WITH CHEST PAIN OF RECENT ONSET}

doi:10.1136/heartjnl-2012-301877b.140

I U Haq, * P C Adams. Royal Victoria Infirmary, Newcastle upon Tyne, UK

Background The NICE guideline for chest pain of recent onset recommends diagnosis of angina based on clinical assessment and estimated likelihood of coronary artery disease (CAD). Pre-test likelihood (PTL) estimates are provided in a simplified table based on the Pryor risk equation. If the PTL of CAD is $<10 \%$ or $>90 \%$, further diagnostic testing is not required. If $10 \%-29 \%$ cardiac CT should be offered, if $30 \%-60 \%$ functional imaging, and if $61 \%-90 \%$ coronary angiography. We compared risk estimation methods to determine how much use of the table in an uncritical manner vs use of the full risk equation led to a different referral pattern. We also investigated whether interpolation for risk factor profiles would improve assessment.

Methods Data were collected prospectively for all patients referred to the Rapid Access Chest Pain Clinic, Newcastle upon Tyne, between February 2002 and August 2011. For each patient, PTL of CAD was assessed by three methods: (1) Reference to the NICE table based on chest pain (typical or atypical), age in deciles, sex and risk factors-smoking, hyperlipidaemia and diabetes dichotomised into high and low risk. For high risk, all three risk factors needed to be present; otherwise the patient was assumed low risk. (2) As per method one, but risk estimates were interpolated between low and high risk values in the NICE table according to the number of risk factors. (3) Calculation by the Pryor equation which includes, in addition, age in years, prior MI, ECG $\mathrm{Q}$ waves, and ST/T changes.

Results Out of an initial 7022 patients, 1820 were excluded as they had non-anginal chest pain. This left 5202 patients, 2889 with atypical angina and 2313 with typical angina. The number (\%) of patients in the five different risk groups by the three risk assessment methods is given in Abstract 140 table 1. Use of the risk equation compared to the table led to half as many people in the category $<10 \%$. Fewer patients would be referred for cardiac CT, more for functional imaging and more for invasive coronary angiography. Use of the adapted table correlated with the risk equation better, but there were still discrepancies, and the percentage of patients for coronary angiography would increase overall (Abstract 140 table 1). The NICE table would classify only $39 \%$ of patients with PTL $<10 \%$ correctly, $60.3 \%$ of those with PTL $10 \%-29 \%, 44.9 \%$ of those with PTL $30 \%-60 \%, 62.9 \%$ of those with PTL $61 \%-90 \%$, and $86.3 \%$ of those with PTL $>90 \%$ (Abstract 140 figure 1). The corresponding figures correctly classifying people in the five risk categories using the adapted table were $92.9 \%, 86.4 \%, 65.2 \%, 71.2 \%$ and $85.9 \%$ respectively (Abstract 140 figure 2).

\section{Abstract 140 Table 1}

\begin{tabular}{llrlll}
\hline $\begin{array}{l}\text { Pre-test } \\
\text { likelihood CAD }\end{array}$ & $<\mathbf{1 0 \%}$ & $\mathbf{1 0 \% - 2 9 \%}$ & $\mathbf{3 0 \% - 6 0 \%}$ & $\mathbf{6 1 \% - 9 0 \%}$ & $\mathbf{> 9 0 \%}$ \\
\hline NICE table-n (\%) & $365(7.0)$ & $1247(24.0)$ & $1069(20.5)$ & $1424(27.4)$ & $1097(21.1)$ \\
Modified table-n (\%) & $127(2.4)$ & $795(15.3)$ & $1271(24.4)$ & $1821(35.0)$ & $1188(22.8)$ \\
Pryor risk & $183(3.5)$ & $1002(19.3)$ & $1190(22.9)$ & $1633(31.4)$ & $1194(23.0)$ \\
equation-n (\%) & & & & & \\
\hline
\end{tabular}

